\section{Diabetes und Adipositas häufiger in sozioökonomisch benachteiligten Regionen}

Menschen, die in sozioökonomisch benachteiligten Regionen leben, sind häufiger von Typ-2-Diabetes und Adipositas betroffen. Dies zeigt eine aktuelle Analyse von Daten der telefonischen Gesundheitsbefragung GEDA durch Wissenschaftler des Helmholtz Zentrums München und des Robert Koch-Instituts in Berlin. Die Ergebnisse sind im Fachjournal PLOS ONE veröffentlicht.

In einer sozioökonomisch benachteiligten Region zu leben, ist ein Risikofaktor dafür, von Diabetes mellitus und Adipositas (Fettleibigkeit) betroffen zu sein. Dies gilt auch unabhängig vom individuellen Sozialstatus der Bewohner. Zu diesem Schluss kommen Wissenschaftler vom Institut für Gesundheitsökonomie und Management im Gesundheitswesen (IGM) am Helmholtz Zentrum München (HMGU) und der Abteilung für Epidemiologie und Gesundheitsmonitoring am Robert Koch-Institut (RKI) in Berlin. „Regionale Faktoren wie das durchschnittliche Einkommen der Bevölkerung, Arbeitslosigkeit oder die Beschaffenheit der Wohnumgebung können alle Bewohner gesundheitlich beeinflussen - unabhängig davon welchen Bildungsstatus die einzelnen Personen innehaben“, erklärt Erstautor Werner Maier.

Unter Federführung von Werner Maier im Team um Dr. Andreas Mielck und Professor Dr. Rolf Holle am HMGU wertete die Autorengruppe Daten von über 33.000 Personen im Alter ab 30 Jahren aus, die 2009 und 2010 an den telefonischen Gesundheitsbefragungen „Gesundheit in Deutschland Aktuell (GEDA)“ des RKI teilgenommen hatten.

In sozioökonomisch benachteiligten Regionen leiden die Bewohner überdurchschnittlich häufig an Diabetes und Übergewicht. Die geographische Benachteiligung wird als „regionale Deprivation“ bezeichnet. Ermittelt wird sie anhand des „German Index of Multiple Deprivation“ (GIMD), der aus regional verfügbaren Informationen zu Einkommen, Beschäftigung, Bildung, kommunalen Einnahmen, Sozialkapital, Umwelt und Sicherheit in einem definierten Gebiet gebildet wird. Neben dem GIMD wurden in der Datenanalyse auch individuelle Risikofaktoren wie Alter, Geschlecht, BodyMass-Index, Rauchen, körperliche Bewegung, Bildung und Leben in einer Partnerschaft berücksichtigt.

In Regionen mit der höchsten Benachteiligung (Deprivation) lag die Häufigkeit eines Typ-2-Diabetes bei 8,6 Prozent der Befragten und für Adipositas bei 16,9 Prozent, gegenüber 5,8 bzw. 13,7 Prozent der Befragten in nur gering benachteiligten (deprivierten) Regionen. Diese Ergebnisse wurden auf relevante Unterschiede in allen individuellen Faktoren überprüft. Demnach hatten Personen in den Gebieten mit der höchsten Deprivation noch eine rund 20 Prozent höhere Wahrscheinlichkeit, an Typ-2-Diabetes erkrankt zu sein, verglichen mit Männern und Frauen in den am wenigsten benachteiligten Regionen. Bei Adipositas lag sogar eine um fast 30 Prozent erhöhte Wahrscheinlichkeit in Zusammenhang mit höherer Deprivation vor. Hohe regionale Deprivation war insbesondere bei Frauen ein unabhängiger Einflussfaktor für das Auftreten von Diabetes und Adipositas. Bei Männern ließ sich ein statistisch signifikanter und unabhängiger Zusammenhang für Adipositas, nicht aber für Diabetes nachweisen.

\author{
Herausgeber \\ Robert Koch-Institut \\ Nordufer 20 \\ D-13353 Berlin \\ www.rki.de \\ Das Robert Koch-Institut \\ ist ein Bundesinstitut im \\ Geschäftsbereich des \\ Bundesministeriums für \\ Gesundheit \\ Pressestelle \\ Susanne Glasmacher \\ (Pressesprecherin) \\ Günther Dettweiler \\ (stellv. Pressesprecher) \\ Heidi Golisch \\ Judith Petschelt

Kontakt
$\begin{array}{ll}\text { Tel.: } & \text { O30-18754-2239, } \\ -2562 \text { und }-2286\end{array}$
Fax: $\quad$ o30-18754 2265
E-Mail: $p$ presse@rki.de
Twitter: @rki_de




\section{Gemeinsame Pressemitteilung \\ Robert Koch-Institut \\ Helmholtz Zentrum München \\ Sperrfrist 28.02.2014,9.00 Uhr}

„Unsere Ergebnisse weisen auf die Bedeutung regionaler Faktoren im Zusammenhang mit häufigen Gesundheitsproblemen wie Diabetes mellitus und Adipositas in Deutschland hin“, erklärt Dr. Andreas Mielck vom HMGU, „bisherige Untersuchungen hierzu waren häufig durch den individuellen sozioökonomischen Status verfälscht oder haben nur regional begrenzte oder nicht aus Deutschland stammende Daten verwendet.“ Werner Maier fügt hinzu: „Räumliche Risikofaktoren wie materielle und soziale Deprivation sind ein wichtiger Ansatzpunkt, um regionalspezifische, effektive Präventionsmaßnahmen zu erarbeiten. “ Dr. Christa Scheidt-Nave vom RKI ergänzt: „Nur Dank großer, bundesweit repräsentativer Befragungen wie GEDA verfügen wir über aktuelle Daten zur Epidemiologie chronischer Krankheiten wie Diabetes. Für ganzheitliche Präventionsstrategien müssen wir regionale wie auch individuelle Risikofaktoren identifizieren und auch deren Interaktion beleuchten."

Von Diabetes mellitus sind nach Ergebnissen des bundesweiten Gesundheitsmonitorings deutschlandweit aktuell rund sechs Millionen Personen über 18 Jahre betroffen, an Adipositas leiden sogar mehr als doppelt so viele Erwachsene.

\section{Weitere Informationen}

Die epidemiologischen Analysen wurden als Teil eines Kooperationsprojektes beider Institutionen zu Diabetes mellitus und sozialer Ungleichheit im Kompetenznetz Diabetes mellitus durchgeführt. Innerhalb dieses Forschungsverbundes werden beide Institutionen durch das Bundesministerium für Bildung und Forschung gefördert. Die Datenerhebungen zu GEDA erfolgen regelmäßig im Rahmen des bundesweiten Gesundheitsmonitorings am RKI mit Förderung des Bundesministeriums für Gesundheit.

Original-Publikation

Werner Maier, Christa Scheidt-Nave, Rolf Holle, Lars E. Kroll, Thomas Lampert, Yong $\mathrm{Du}$, Christin Heidemann, Andreas Mielck (2014), Area level deprivation is an independent determinant of prevalent type 2 diabetes and obesity at the national level in Germany. Results from the national telephone health interview surveys 'German Health Update' GEDA 2009 and 2010, PLOS ONE, doi: pone.0089661

Link zur Fachpublikation: http://dx.plos.org/10.1371/journal.pone.0089661

Das Helmholtz Zentrum München verfolgt als Deutsches Forschungszentrum für Gesundheit und Umwelt das Ziel, personalisierte Medizin für die Diagnose, Therapie und Prävention weit verbreiteter Volkskrankheiten wie Diabetes mellitus und Lungenerkrankungen zu entwickeln. Dafür untersucht es das Zusammenwirken von Genetik, Umweltfaktoren und Lebensstil. Der Hauptsitz des Zentrums liegt in Neuherberg im Norden Münchens. Das Helmholtz Zentrum München beschäftigt rund 2.200 Mitarbeiter und ist Mitglied der Helmholtz-Gemeinschaft, der 18 naturwissenschaftlich-technische und medizinisch-biologische Forschungszentren mit rund $34.000 \mathrm{Be}-$ schäftigten angehören. www.helmholtz-muenchen.de 
Das Institut für Gesundheitsökonomie und Management im Gesundheitswesen (IGM) untersucht Ansätze zur Verbesserung der Wirksamkeit und Wirtschaftlichkeit der Gesundheitsversorgung. Das Gesundheitssystem steht vor der Herausforderung, allen Bürgern eine qualitativ hochwertige und finanziell tragbare medizinische Versorgung zu gewährleisten. Der rasche medizinisch-technische Fortschritt und die demographischen Veränderungen verschärfen das beschriebene Spannungsverhältnis. Eine fundierte Evaluation der Strukturen und Prozesse der gesundheitlichen Versorgung unter den Gesichtspunkten von Effektivität und Effizienz ist eine unabdingbare Voraussetzung für rationales Handeln.

Das Robert Koch-Institut ist das nationale Public-Health-Institut. Als wissenschaftlichmedizinische Leitinstitution der Bundesregierung spielt es bei der Vorbeugung und Bekämpfung von Infektionskrankheiten und der Analyse langfristiger gesundheitlicher Trends in der Bevölkerung eine herausragende Rolle im deutschen Gesundheitswesen. 